\title{
Pérdidas Joule en alimentadores que transportan corrientes armónicas en baja tensión
}

\author{
Joule losses in feeders that transport harmonic currents in low voltage \\ Guillermo F. di Mauro ${ }^{1} \quad$ Juan A. Suárez ${ }^{1} \quad$ Daniel O. Anaut ${ }^{1}$ \\ Rubén R. di Mauro $^{1} \quad$ Carlos A. Agüero ${ }^{1}$ \\ Recibido 9 de junio de 2011, aceptado 20 de mayo de 2013 \\ Received: June 9, 2011 Accepted: May 20, 2013
}

\begin{abstract}
RESUMEN
Se presenta un estudio de las pérdidas Joule en alimentadores que conducen corrientes no sinusoidales. Distintas fórmulas propuestas en la literatura fueron utilizadas para la estimación de la corriente de neutro y de las pérdidas producidas. En el entorno del Alternative Transients Program, se simularon cargas con diferentes niveles de distorsión armónica, arribando a resultados que demuestran que las pérdidas pueden llegar a ser considerablemente superiores a la condición en la que se transporta la misma potencia activa sin distorsión. Se analiza un caso real y la importancia de la reducción del contenido de armónicos asociados a las pérdidas eléctricas en líneas de alimentación.
\end{abstract}

Palabras clave: Sistemas de distribución, pérdidas Joule, pérdidas en alimentadores, armónicos, corrientes de neutro, cargas no lineales.

\begin{abstract}
A study of Joule losses in electrical feeder lines carrying harmonic currents is presented. Different formulae proposed in the literature were used to estimate the neutral current and electrical losses. In the environment of the Alternative Transients Program, different levels of harmonic distortion were simulated, arriving at findings that show that losses may become considerably higher than in the condition in which active power without distortion is carried. A real case and the importance of reducing the harmonic content associated with losses are analyzed.
\end{abstract}

Keywords: Power distribution, Joule losses, losses on feeders, harmonics, neutral current, nonlinear load.

\section{INTRODUCCIÓN}

El uso cada vez más frecuente de cargas no lineales como computadoras personales, lámparas fluorescentes compactas, fuentes conmutadas de diferentes equipos electrónicos, etc., ha provocado una constante degradación de la calidad de la energía en los sistemas de distribución eléctrica, manifestado por la aparición de componentes armónicos de tensión y de corriente [1].
La presencia de armónicos en la red trae aparejado diversos inconvenientes tanto en los equipos conectados como a los propios componentes [2] tales como: pérdidas adicionales en máquinas y transformadores, calentamiento en capacitores, fallos en equipamiento de protección, distorsión en la medición de energía, aumento en las corrientes de neutro, incremento de pérdidas en conductores, sobredimensionamiento de instalaciones, etcétera.

\footnotetext{
1 Departamento de Ingeniería Eléctrica. Universidad Nacional de Mar del Plata. Juan B. Justo 4302, Mar del Plata, CP 7600, Pcia. Buenos Aires, Argentina. E-mail: gdimauro@copetel.com.ar; jsuarez@fi.mdp.edu.ar; danaut@ fi.mdp.edu.ar; rdimauro@fi.mdp.edu.ar; caguero@fi.mdp.edu.ar
} 
En algunos países existen normas que limitan la producción de armónicos, sin embargo, se encuentran instalaciones cuya generación de corrientes armónicas están muy por encima de los límites aceptados. Estudios realizados en Estados Unidos revelaron que más del $20 \%$ de los complejos comerciales tienen en sus instalaciones corrientes de neutro superiores a las de línea [3], evidenciando fuertes contenidos de terceros armónicos y sus múltiplos.

La situación planteada tiene un costo significativo para las compañías distribuidoras, hasta el punto de que en algunos países se está considerando la creación de un recargo sobre la tarifa de consumo para compensar económicamente la infraestructura de transporte y las pérdidas adicionales [4].

En este contexto, el trabajo propone analizar las pérdidas por efecto Joule en alimentadores que suministran energía eléctrica a cargas no sinusoidales. El Alternative Transients Program (ATP) fue utilizado para simular condiciones de cargas con distintos niveles de distorsión. A partir de los resultados de las simulaciones, distintas fórmulas propuestas en la literatura [1, 5-7] fueron utilizadas para la estimación de la corriente de neutro y el cálculo de las pérdidas Joule en relación con las mínimas pérdida de potencia en la línea, para el mismo valor de potencia activa transmitida.

\section{REVISIÓN TEÓRICA}

\section{Cálculo de la corriente de neutro}

En un sistema eléctrico trifásico, la corriente de neutro es el vector suma de las tres corrientes de líneas. Si el sistema de alimentación es balanceado, con una simetría de sus ondas en $120^{\circ}$ eléctricos y con carga lineal trifásica perfectamente balanceada, la corriente de neutro es igual a cero. En la práctica se espera que en un sistema trifásico de cuatro hilos medianamente balanceado, la corriente que circula por el neutro sea apenas $20 \%$ de la corriente de fase. Muy distinto es el escenario si las cargas no son lineales. La aparición de los componentes armónicos impares, en particular los múltiplos de tres, inciden en el aumento de la corriente en el neutro aunque el sistema se encuentre balanceado. En un sistema trifásico de cuatro hilos con cargas no lineales balanceadas, la corriente en el neutro dependerá básicamente del contenido armónico de las corrientes de fases.

Si se suponen cargas no lineales con componentes armónicos impares, se puede descomponer a cada una de las corrientes de fase usando la transformada de Fourier [1]:

$$
\begin{gathered}
i_{R}=\hat{I}_{1} \operatorname{sen}\left(w t+\alpha_{1}\right)+\hat{I}_{3} \operatorname{sen}(3 w t+. . \\
\left.. .+\alpha_{3}\right)+\hat{I}_{5} \operatorname{sen}\left(5 w t+\alpha_{5}\right)+. . \\
i_{S}=\hat{I}_{1} \operatorname{sen}\left(w t-\frac{2 \pi}{3}+\alpha_{1}\right)+\hat{I}_{3} \operatorname{sen}(3(\omega t-. . \\
\left.\left.. .-\frac{2 \pi}{3}+\alpha_{3}\right)\right)+\hat{I}_{5} \operatorname{sen}\left(5\left(w t-\frac{2 \pi}{3}+\alpha_{5}\right) . .\right. \\
i_{T}=\hat{I}_{1} \operatorname{sen}\left(w t-\frac{4 \pi}{3}+\alpha_{1}\right)+\hat{I}_{3} \operatorname{sen}(3(\omega t-. . \\
\left.\left.. .-\frac{4 \pi}{3}+\alpha_{3}\right)\right)+\hat{I}_{5} \operatorname{sen}\left(5\left(w t-\frac{4 \pi}{3}+\alpha_{5}\right) . .\right.
\end{gathered}
$$

Donde:

$i_{R}, i_{S}, i_{T}$ : valor instantáneo de las corrientes de las fases R, S y T.

$\hat{I}_{1}, \hat{I}_{3}, \hat{I}_{5}$ : amplitud de las corrientes armónicas 1, 3, 5 . $\alpha_{1}, \alpha_{3}, \alpha_{5}$ : ángulo de fase de la corriente armónica $1,3,5$.

La expresión de la corriente en el neutro en un sistema trifásico simétrico y balanceado puede ser escrita como la sumatoria de las corrientes de las fases:

$$
\begin{aligned}
& i_{N}=i_{R}+i_{S}+i_{T}=\ldots \\
& . .=\Sigma 3 \hat{I}_{6 k+3} \operatorname{sen}\left((6 k+3) w t+\alpha_{6 k+3}\right)
\end{aligned}
$$

Donde:

$k: 0,1,2,3 \ldots$

La ecuación (4) muestra que la corriente en el neutro, bajo condiciones balanceadas, es producida por las componentes de secuencia cero $(3,9,15,21 \ldots)$ de las corrientes de fases.

En un circuito simétrico y balanceado, la relación entre la corriente de neutro y la de fase se incrementa con el aumento del armónico de tercer orden y con la disminución del primero y quinto de la corriente de fase, según se demuestra en [6], donde se define: 


$$
\begin{aligned}
& \rho_{1}=\frac{I_{N}}{I_{f}}=\ldots \\
& . . \frac{\sqrt{\sum\left(3 I_{6 k+3}\right)^{2}}}{\sqrt{\sum\left(3 I_{6 k+1}\right)^{2}+\sum\left(3 I_{6 k+3}\right)^{2}+\sum\left(3 I_{6 k+5}\right)^{2}}}
\end{aligned}
$$

Donde:

$I_{N}$ : valor rms de la corriente total del neutro. $I_{f}$ : valor rms de la corriente total de fase.

$I_{6 k+1}, I_{6 k+3}, I_{6 k+5}$ : valor rms de la corriente armónica de orden $6 \mathrm{k}+1,6 \mathrm{k}+3,6 \mathrm{k}+5(\mathrm{k}=0,1,2 .$.$) .$

Si las corrientes de fases solo tienen componentes armónicos impares $\mathrm{I}_{2 \mathrm{n}+1}$ con $\mathrm{I}_{2 \mathrm{n}+1}=\mathrm{q}^{\mathrm{n}} \mathrm{I}_{1}$, con $0 \leq \mathrm{q}$ $\leq 1$ y $n=1,2,3$. . $\left(I_{3}=q \cdot I_{1}, I_{5}=q^{2} \cdot I_{1}, I_{7}=q^{3} \cdot I_{1} \ldots\right)$, se deduce que la relación entre la corriente de neutro y fase resulta ser [6]:

$$
\rho_{1}=3 \frac{q}{\sqrt{1+q^{2}+q^{4}}}
$$

La corriente de neutro es máxima e igual a $\sqrt{3}$ veces la corriente de línea cuando $\mathrm{q}=1$, condición que se cumple cuando todos los componentes armónicos tienen el mismo peso.

La fuerte relación entre la tercera armónica y la corriente de neutro conduce a una igual relación entre esta última y la distorsión armónica de la corriente de fase (THD). Así, expresando la componente fundamental $\left(\mathrm{I}_{1}\right)$ y la de tercer armónico $\left(\mathrm{I}_{3}\right)$ en p.u. de la corriente rms total de fase, según [5], puede representarse por:

$$
T H D>\frac{\sqrt{I_{3}^{2}}}{I_{1}}=\frac{I_{3}}{I_{1}}
$$

Dado que $\mathrm{I}_{1}$ e $\mathrm{I}_{3}$ están expresadas en p.u. del valor rms de la corriente de fase y dado que $I_{1}>I_{3}$, surge que:

$$
\sqrt{I_{1}^{2}+I_{3}^{2}}<1 \text { о } I_{1}<\sqrt{1-I_{3}^{2}}
$$

Combinando las ecuaciones (7) y (8) resulta:

$$
I_{3}<\frac{T H D}{\sqrt{1+T H D^{2}}}
$$

Dado que los armónicos superiores al tercero no tienen un significante aporte a la corriente de neutro:

$$
I_{N} \cong 3 I_{3}
$$

Combinando las ecuaciones (9) y (10) resulta que la relación entre la corriente de neutro y fase puede volver a escribirse como:

$$
\rho_{2}=\frac{I_{N}}{I_{f}}=I_{N}=3 \frac{T H D}{\sqrt{1+T H D^{2}}}
$$

La ecuación (11) es válida bajo el supuesto que los armónicos son impares y el de tercer orden es el más preponderante. Alcanza el máximo $(1,73)$ cuando el índice de distorsión de corriente es del 70,7\%.

\section{Cálculo de las pérdidas por efecto Joule en alimentadores}

Suponiendo que los conductores de línea y el neutro tienen el mismo valor de resistencia $\left(R_{L}\right)$, las pérdidas por efecto Joule en aquellas pueden ser calculadas como:

$$
P_{L}=\left(I_{R}^{2}+I_{S}^{2}+I_{T}^{2}+I_{N}^{2}\right) R_{L}
$$

Utilizando las definiciones de potencia en redes trifásicas propuestas en la bibliografía [10], de donde surge la expresión de la corriente equivalente $\left(I_{e}\right)$ :

$$
I_{e}^{2}=\frac{\left(I_{R}^{2}+I_{S}^{2}+I_{T}^{2}+I_{N}^{2}\right)}{3}
$$

La ecuación (12) se puede escribir como:

$$
P_{L}=3 I_{e}^{2} R_{L}
$$

En función de que la corriente de neutro sea calculada en forma exacta (a partir de los componentes armónicos de las corrientes de fase) o hallada utilizando la relación $\rho_{1} \circ \rho_{2}$, resultarán diferentes estimaciones de las pérdidas en los alimentadores.

\section{Cálculo de las pérdidas mínimas por efecto Joule en alimentadores}

De acuerdo con las expresiones propuestas en [7], las pérdidas mínimas de potencia en la línea $\left(P_{L m n}\right)$, para un mismo valor de potencia activa transmitida a la carga, puede calcularse con la expresión: 


$$
P_{L m n}=\left(I_{R}^{2}+I_{S}^{2}+I_{T}^{2}\right) R_{L}
$$

Si las corrientes $I_{R}, I_{S}, I_{T}$, son sinusoidales y en fase con la componente directa de la tensión, se cumple la condición de pérdidas mínimas con tensiones de alimentación sinusoidal y equilibrada.

La potencia demandada en la carga, en régimen de pérdidas mínimas es:

$$
P_{1}=3 V_{1} I_{1} \cos \varphi_{1}
$$

Las pérdidas mínimas en esta condición, son:

$$
P_{L m n}=3 I_{1}^{2} \cos ^{2} \phi_{1} R_{L}
$$

Dividiendo miembro a miembro (17) y (14), resulta:

$$
\frac{P_{L m n}}{P_{L}}=\frac{I_{1}^{2} \cos ^{2} \varphi_{1}}{I_{e}^{2}}
$$

Al igual que la ecuación (13), se define la tensión equivalente $\left(V_{e}\right)$ como [10]:

$$
V_{e}^{2}=\frac{\left(V_{R}^{2}+V_{S}^{2}+V_{T}^{2}\right)}{3}
$$

con lo cual la ecuación (18) se puede expresar como:

$$
\frac{P_{L m n}}{P_{L}}=\frac{S_{1} \cos \varphi_{1}}{S_{e}}\left[\frac{V_{E}}{V_{1}}\right]^{2}
$$

Donde:

$S_{e}=I_{e} \cdot V_{e}$

Siendo $S_{1} \cos \varphi_{1} / S_{e}$, la relación entre la potencia de componente directa y la potencia aparente equivalente, que se designa $F P_{l}$ (Factor de Potencia de Componente Directa).

$$
\frac{P_{L m n}}{P_{L}}=F P_{1}^{2}\left[\frac{V_{e}}{V_{1}}\right]^{2}
$$

Admitiendo que en la mayoría de los casos $\mathrm{V}_{\mathrm{e}} \cong$ $\mathrm{V}_{1}$, resulta:

$$
\frac{P_{L m n}}{P_{L}} \cong F P_{1}^{2}
$$

\section{SIMULACIÓN DE CASO Y RESULTADOS}

En el entorno del ATP se simularon diferentes condiciones de carga con distintos niveles de distorsión. Para ello fue utilizado el circuito de la Figura 1, en donde se representa un sistema trifásico con neutro, alimentando a fuentes rectificadoras monofásicas tipo puente con cargas resistivas (Figura 2). La alimentación y la carga son simétricas y balanceadas.

Las fuentes de tensión son del tipo 14 y ajustadas a 220 Vrms. Se supuso que las características eléctricas de los alimentadores de fase y neutro son iguales, con parámetros:

$\mathrm{R}_{\mathrm{L}}=0,2$ Ohm y $\mathrm{L}_{\mathrm{L}}=0,04 \mathrm{mHy}$.

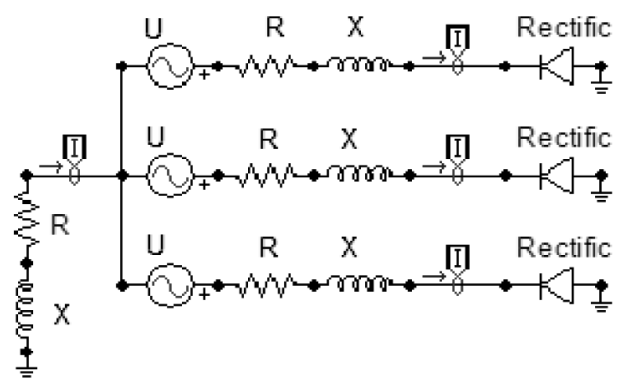

Figura 1. Circuito trifásico.

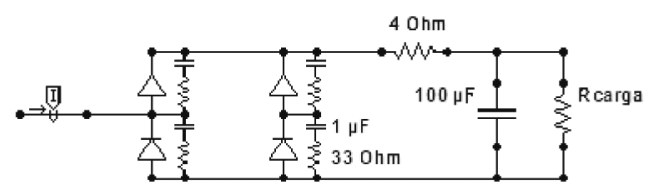

Figura 2. Modelo de rectificador tipo puente.

Por variación de la resistencia de carga de las fuentes rectificadoras se logra una alteración de la composición de armónicos en relación con la fundamental. Con ello se obtienen diferentes valores de la distorsión armónica de la corriente de alimentación (THD) manteniendo el cos $\varphi$ de la componente fundamental en 0,93, aproximadamente. La Tabla 1 muestra, para cada THD logrado, la composición de armónicos relacionada. 
Tabla 1. Relación porcentual entre la corriente de cada componente armónica y la fundamental.

\begin{tabular}{|c|c|c|c|c|c|c|c|c|c|c|}
\cline { 2 - 12 } \multicolumn{1}{c|}{} & \multicolumn{10}{c|}{ THD\% } \\
\hline$[\mathbf{H z}]$ & $\mathbf{2 6 , 2}$ & $\mathbf{3 9 , 5}$ & $\mathbf{4 9 , 3}$ & $\mathbf{5 3 , 2}$ & $\mathbf{5 6 , 6}$ & $\mathbf{6 2 , 6}$ & $\mathbf{7 2 , 7}$ & $\mathbf{8 0 , 3}$ & $\mathbf{9 4 , 5}$ & $\mathbf{1 0 5 , 0}$ \\
\hline $\mathbf{5 0}$ & 100 & 100 & 100 & 100 & 100 & 100 & 100 & 100 & 100 & 100 \\
\hline $\mathbf{1 5 0}$ & 20,3 & 35,3 & 45,6 & 49,5 & 52,7 & 58,0 & 65,7 & 68,5 & 76,6 & 81,6 \\
\hline $\mathbf{2 5 0}$ & 14,4 & 13,4 & 10,2 & 9,8 & 10,8 & 15,2 & 25,3 & 32,5 & 45,7 & 54,6 \\
\hline $\mathbf{3 5 0}$ & 5,9 & 9,3 & 13,8 & 147 & 14,9 & 14,5 & 12,8 & 19,1 & 22,7 & 28,3 \\
\hline $\mathbf{4 5 0}$ & 2,5 & 5,0 & 4,0 & 3,9 & 4,5 & 6,5 & 9,5 & 12,7 & 14,1 & 14,6 \\
\hline $\mathbf{5 5 0}$ & 4,0 & 2,8 & 4,6 & 5,8 & 6,2 & 5,8 & 3,6 & 6,4 & 11,1 & 13,4 \\
\hline $\mathbf{6 5 0}$ & 2,5 & 3,6 & 3,4 & 3,3 & 4,0 & 5,7 & 6,3 & 8,8 & 8,6 & 10,6 \\
\hline $\mathbf{7 5 0}$ & 0,7 & 1,6 & 1,7 & 2,2 & 2,3 & 1,8 & 2,3 & 3,9 & 5,0 & 5,8 \\
\hline $\mathbf{8 5 0}$ & 1,7 & 1,1 & 2,3 & 1,7 & 1,4 & 2,6 & 3,2 & 4,1 & 3,6 & 4,3 \\
\hline $\mathbf{9 5 0}$ & 1,4 & 1,8 & 1,7 & 2,3 & 2,3 & 1,7 & 3,2 & 5,4 & 4,3 & 4,4 \\
\hline $\mathbf{1 0 5 0}$ & 0,3 & 0,6 & 1,0 & 0,7 & 0,7 & 1,2 & 0,9 & 1,2 & 2,0 & 2,8 \\
\hline
\end{tabular}

Para los diferentes valores logrados de THD de la corriente de alimentación y en base a las fórmulas planteadas en el título anterior se hallaron las corrientes de neutro y las pérdidas Joule provocadas por la circulación de corrientes en las fases y en el neutro.

La Figura 3 muestra la relación entre las corrientes de neutro y de fase, medidas en el esquema de simulación y las estimadas a partir de las expresiones de $\rho_{1}$ y de $\rho_{2}$.

Por otro lado, en base a la misma carga del ejemplo simulado se ha calculado la relación entre la corriente de neutro y de fase para una condición de distorsión de corriente (THD=25,5\%) y distribución de armónicos (Tabla 2) que fija el Ente Nacional Regulador de la Electricidad de Argentina (ENRE) [8] que se muestra en la misma Figura 3.

La relación entre las pérdidas Joule en los alimentadores (originadas para cada condición de THD) y las mínimas pérdidas para las mismas condiciones de carga, fue calculada aplicando las relaciones antes descriptas:

Relación entre valores de Pérdidas reales en la Línea y Pérdidas Mínimas:

$$
R_{r}=P_{\text {real }} / P_{\text {Lmn }}
$$

Relación entre Pérdidas en la Línea estimada con $\rho_{1}$ y Pérdidas Mínimas:
Tabla 2. Distribución de Armónicos ENRE.

\begin{tabular}{|c|c|}
\hline$[\mathrm{Hz}]$ & $\% \mathrm{I}_{1}$ \\
\hline 50 & 100 \\
\hline 150 & 16,6 \\
\hline 250 & 12,0 \\
\hline 350 & 8,5 \\
\hline 450 & 2,2 \\
\hline 550 & 4,3 \\
\hline 650 & 3,0 \\
\hline 750 & 0,6 \\
\hline 850 & 2,7 \\
\hline 950 & 1,9 \\
\hline 1050 & 0,4 \\
\hline
\end{tabular}

$$
R_{\rho_{1}}=P \rho_{1} / P_{L m n}
$$

Relación entre Pérdidas en la Línea estimada con 2 y Pérdidas Mínimas:

$$
R_{\rho_{2}}=P \rho_{2} / P_{L m n}
$$

Relación entre Pérdidas en la Línea y Pérdidas Mínimas estimada a partir de $\mathrm{FP}_{1}^{2}$ :

$$
R_{f p}=1 / F P_{1}^{2}
$$

También fue hallada la relación entre las Pérdidas en la Línea y Pérdidas Mínimas para el caso de transportar una corriente en condiciones de distorsión ENRE (punto $R_{\text {enre }}$ ), por medio de cálculos, sin estimación. 


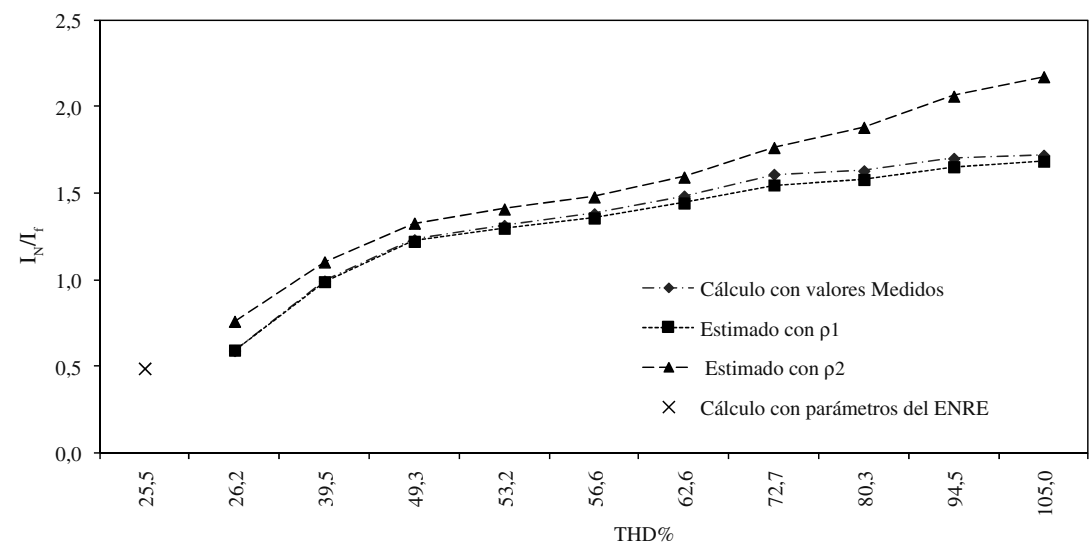

Figura 3. $\mathrm{I}_{\mathrm{N}} / \mathrm{I}_{\mathrm{f}}$ en función de la distorsión de corriente.

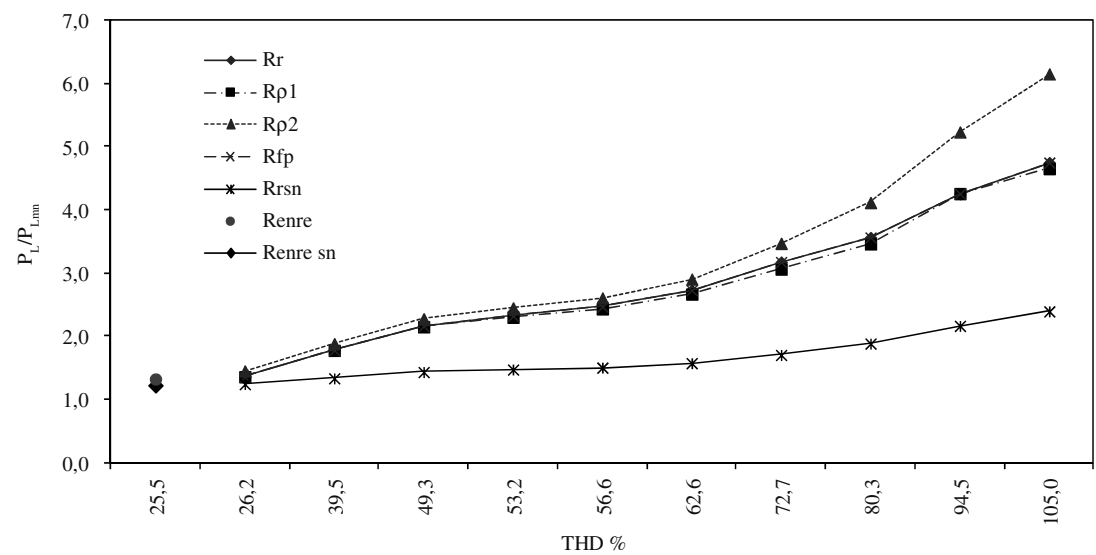

Figura 4. Relación entre pérdidas en condición de carga normal y pérdidas mínimas.

La Figura 4 grafica el resultado de las expresiones anteriores.

En la figura mencionada se muestra la curva de la misma relación de pérdidas reales, pero considerando solo las pérdidas en los conductores de fase (curva $\left.R_{r s n}\right)$. El mismo cálculo fue realizado para la condiciones de distorsión ENRE (punto $R_{\text {enre sn }}$ ).

\section{DISCUSIÓN}

A los efectos del cálculo de las pérdidas en alimentadores, en este trabajo, no se tuvo en cuenta el efecto skin o pelicular, concentrándose en el incremento de pérdidas por aumento de la circulación de corriente por el neutro del circuito. El fenómeno de redistribución de cargas en la periferia de los conductores puede ser perceptible a frecuencias superiores a $300 \mathrm{~Hz}$. Dependiendo del contenido de armónicos de las corrientes y las características constructivas de los conductores, puede considerarse hasta $13 \%$ de incremento en las pérdidas [2]. Trabajos similares reportan incrementos de pérdidas por efecto pelicular y de proximidad del orden del 1,5\% para los casos de estudio [9].

De acuerdo con la Figura 3, en la que se muestra la relación entre la corriente de neutro y de fase, el límite de la misma tiende a 1,73, hecho que se corrobora a partir de la medición de la $\mathrm{I}_{\mathrm{f}} \mathrm{e} \mathrm{I}_{\mathrm{N}}$ en el circuito simulado. La relación $\rho_{1}$ (ecuación (6)), resulta una buena aproximación introduciendo en la misma el valor de q como $\mathrm{I}_{3} / \mathrm{I}_{1}$ para cada caso. La relación expresada por $\rho_{2}$ (ecuación (11)) no es válida más allá de un THD del 70,7\%, corroborándose en la Figura 3 por un apartamiento de la curva de los valores reales, incluso superando el límite de 1,73 de la relación. 
Puede observarse en la Figura 4 el incremento de las pérdidas en los alimentadores con el aumento de la distorsión armónica de corriente de fase (THD). Esta dependencia está fuertemente relacionada con la circulación de corrientes por el neutro. En efecto, los armónicos de tercer orden y sus múltiplos circulantes en una fase se multiplican por tres en su circulación por el conductor de retorno que, al elevarlo al cuadrado, incrementan las pérdidas en el mismo. En la misma figura la curva demarcada con $\mathrm{R}_{\mathrm{rsn}}$ muestra cuáles serían las pérdidas sin tener en cuenta dicha circulación por el neutro. Por ejemplo, si consideramos como la unidad (1) a las pérdidas que por efecto Joule se desarrollan en los alimentadores en la condición de transportar solo la potencia activa, vemos que para un THD de 56,6\% las pérdidas solo en los conductores de fase toman un valor de 1,5 (curva $R_{\mathrm{rsn}}$ ), mientras que las totales, incluyendo a las del conductor neutro, ascienden a 2,46 (curva $\mathrm{R}_{\mathrm{r}}$ ). En este caso, el no considerar las pérdidas en el neutro llevaría a un error del $40 \%$ en defecto. Realizando el mismo análisis para la condición de distorsión ENRE, con un THD de $25,5 \%$, el error sería del $8 \%$ (puntos demarcados con $R_{\text {enre }}$ y $R_{\text {enre sn }}$, Figura 4).

Como se había mencionado anteriormente, en el circuito de ensayo se consideró el mismo tipo de conductor para la fase y el neutro. Si se tiene en cuenta que es común que la sección del conductor neutro sea el 50\% de la de fase, las pérdidas en el neutro se verían incrementadas en el mismo orden de la reducción, haciendo más notable las diferencias mostradas en la Figura 4.

\section{CASO REAL}

Con el fin de evaluar las pérdidas producidas por corrientes armónicas en un sistema de alimentación real, se realizaron mediciones de magnitudes de corrientes en alimentadores de un centro de cómputos de una empresa prestadora de servicios públicos.

Las magnitudes armónicas medidas con un analizador de redes, asociadas a la corriente de una fase, son las que se muestran en la Figura 5, siendo de 81,26\% el THD de corriente de la misma.

Considerando el equilibrio de la carga, la corriente de circulación por el neutro resulta de 1,5 veces mayor que la de fase.
Realizando un análisis de las pérdidas en los alimentadores para el espectro de frecuencias mostrado en la Figura 5 (tomando como $100 \%$ a la suma de las pérdidas provocadas por las corrientes de las tres fases más la del neutro), resulta que las producidas por el armónico fundamental son del $34,3 \%$, las producidas por los armónicos superiores a 1 son del 22,6\%, mientras que la circulación de los armónicos por el neutro aportan $43 \%$.

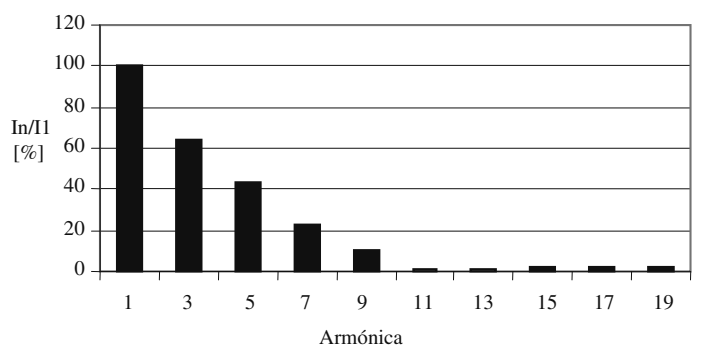

Figura 5. Componentes armónicos de la corriente de fase como porcentaje de la fundamental.

\section{CONCLUSIONES}

De acuerdo con los gráficos anteriores puede deducirse que la ecuación presentada en [6] " $\rho_{1}$ ", constituye una buena aproximación para la estimación de la corriente de neutro para un circuito dentro de un campo de amplia variación del THD. No ocurre lo mismo con el uso de " $\rho_{2}$ " presentada por [5], pues su apartamiento de los valores reales se manifiestan por encima de un THD de 70,7\% y se demuestra en la Figura 3. A la misma inferencia se llega con la utilización de las ecuaciones para el cálculo de las relaciones entre las pérdidas en condición de carga normal y de pérdidas mínimas. Sí resulta una buena aproximación para el cálculo de esta relación, la utilización de las ecuaciones planteadas por [7] en función del Factor de Potencia de Componente Directa (ecuación (21)).

De acuerdo con los resultados obtenidos, y en condiciones de elevados valores de THD, las pérdidas totales en los alimentadores pueden llegar a ser considerablemente superiores a aquella condición en la que se transporta la misma potencia activa sin distorsión, siendo el mayor aporte de este incremento las producidas en el conductor neutro.

El estudio de las mediciones aportadas por la toma de corrientes de un centro de cómputos arroja una 
clara evidencia de la importancia que adquiere la circulación de corriente por el neutro, a causa de las componentes de tercer orden y sus múltiplos, en el aporte a las pérdidas totales en los alimentadores.

A partir de estas apreciaciones se concluye en la importancia de la reducción del contenido de armónicos en líneas de alimentación bajo este punto de vista, no siempre tenido en cuenta y más allá de los perjuicios mencionados anteriormente.

\section{REFERENCIAS}

[1] J. Suárez, G. di Mauro, D. Anaut y R. di Mauro. "Parámetros que Afectan la Corriente de Neutro en Presencia de Armónicos". Revista Información Tecnológica. Vol. $21 \mathrm{~N}^{\circ} 1$. Enero-Febrero de 2010. ISSN 0718-0764.

[2] M. Brugnoni. "Efecto de las Cargas Distorsivas en las Redes de Distribución Eléctrica”. Duodécimo Encuentro Regional Iberoamericano del CIGRÉ-XII ERIAC. Foz Do Iguazú, Brasil. Mayo de 2007.

[3] H.L. Jou, J. Wu, K. Wu, W. Chiang and Y. Chen. "Analysis of zig-zag transformers applayng in the thee-phase for wire distribution power system". IEEE Transaction On Power Delivery. Vol. 20, Issue 2, pp. 1168-1173. 2005.

[4] J. Balcells. "El Coste de los Armónicos". Interempresas net. Septiembre 2004. Fecha de consulta: 4 de marzo de 2011. URL: http:// www.interempresas.net/Plastico/Articulos/ Articulo.asp?A=9294
[5] R. Arthur and R.A. Shanahan. "Neutral Currents in Three Phase wye Systems". IEEE, 7th. International Conference on Harmonics and Quality of Power. Las Vegas, USA. 16 al 18 de Octubre 1996.

[6] J. Desmet, I. Sweertvaegher, G. Vanalme, K. Stockman and R. Belmans. "Analysis of the neutral conductor current in a three phase supplied network with non-linear single phase loads". IEEE Transactions on Industry Applications. Vol. 39, Issue 3, pp. 587-593. 2003.

[7] L.I. Eguíluz, J.C. Lavandero y M. Mañana. "Criterios para una facturación de la energía eléctrica más equitativa”. Revista Energía: Ingeniería Energética y Medioambiental. Año No $28 \mathrm{~N}^{\mathrm{o}} 165$, pp. 125-128. 2002. ISSN 0210-056.

[8] Ente Nacional Regulador de la Electricidad (Argentina). "Base Metodológica para el Control de la Emisión de Perturbaciones". Resolución Nº 99/97.

[9] F.L. Tofoli, S.M. Rivera Sanhueza and A. de Olivera. "On the Study of Losses in Cables and Transformers in Nonsinusoidal Conditions". IEEE Transactions on Power Delivery. Vol. 21, Issue 2, pp. 971-978. April, 2006.

[10] A.E. Emanuel. "Summary of IEEE Standard 1459: Definitions for the Measurement of Electric Power Quantities Under Sinusoidal, Nonsinusoidal, Balanced, or Unbalanced Conditions". IEEE Transactions on Industry Applications. Vol. 40, Issue 3, pp. 869-876. May-June, 2004. 